\title{
Resolving the structure of the Galactic foreground using Herschel measurements and the Kriging technique
}

\author{
S. Pinter ${ }^{1}$, Z. Bagoly ${ }^{1}$, L. G. Balázs ${ }^{1,2}$, I. Horvath ${ }^{3}$, I. I. Racz ${ }^{1,2}$, \\ S. Zahorecz ${ }^{4,5}$ and L. V. Tóth ${ }^{1,2}$ \\ ${ }^{1}$ Eötvös University, Budapest, email: s.pinter@astro.elte.hu \\ ${ }^{2}$ MTA CSFK Konkoly Observatory, Budapest; ${ }^{3}$ National University of Public Service, \\ Budapest; ${ }^{4}$ Osaka Prefecture University, Sakai; ${ }^{5}$ National Astronomical Observatory \\ of Japan, Mitaka-shi
}

\begin{abstract}
Investigating the distant extragalactic Universe requires a subtraction of the Galactic foreground. One of the major difficulties deriving the fine structure of the galactic foreground is the embedded foreground and background point sources appearing in the given fields. It is especially so in the infrared. We report our study subtracting point sources from Herschel images with Kriging, an interpolation method where the interpolated values are modelled by a Gaussian process governed by prior covariances. Using the Kriging method on Herschel multi-wavelength observations the structure of the Galactic foreground can be studied with much higher resolution than previously, leading to a better foreground subtraction at the end.
\end{abstract}

Keywords. ISM: structure, infrared: ISM, infrared: stars, techniques: image processing

\section{Introduction}

To measure the physical parameters of the interstellar medium (ISM) from far infrared (FIR) observations we need to remove the contamination originate from both the instrument (e.g. instrumental, detection, and processing errors) and the irrelevant signals in the observations (e.g. point sources or extended sources). In our work we demonstrate the usage of Kriging (Gaussian process regression or Wiener-Kolmogorov prediction) in determining the properties of ISM, a method which is widely used in several areas (e.g. geostatistics, climatology) but slightly underused in astronomy.

The idea of Kriging is to predict the value of a function at a given point by computing a weighted average of the known values of the function in the neighborhood of the point. It derive a best linear unbiased estimator, based on assumptions on covariances, make use of Gauss-Markov theorem to prove independence of the estimate and error.

We demonstrate the efficiency of the Kriging on a medium Galactic latitude region towards the Taurus molecular clouds where the nearby clumpy clouds of Taurus challenge the point source subtraction with its complexity.

\section{Analysis and results}

As part of the Computer Aided Data Processing Project of the HEART $\dagger$ we recently developed the ADWO software package [Bagoly et al. (2016)] and a Kriging software tool (see this report). For our study we selected the TMC-1 field of the Taurus which is a well-studied nearby star forming the area [Fehér et al. (2016)]. 100 artificial sources with

$\dagger$ High Energy Astronomy Research Team, http://www.konkoly.hu/HEART/ 


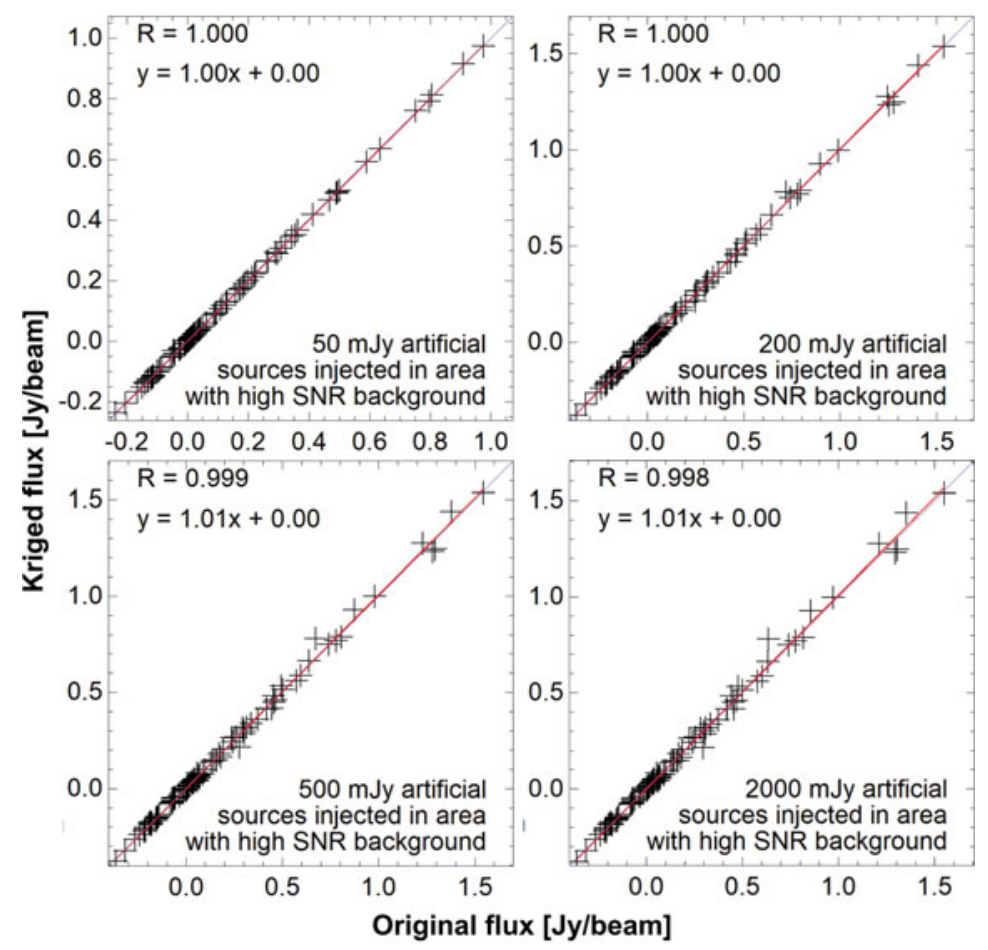

Figure 1. The goodness of the point source removal on the TMC-1 field of the Taurus. The charts show the sum flux of a beam sized area before insertation (original) and after subtraction (kriged) with inserted fluxes of $50 \mathrm{mJy}$ (top left), $200 \mathrm{mJy}$ (top right), $500 \mathrm{mJy}$ (bottom left), and $2000 \mathrm{mJy}$ (bottom right).

different fluxes were inserted onto Herschel SPIRE FIR observations, than were removed with Kriging technique. Fig. 1 shows the goodness of the removal in the $250 \mu \mathrm{m}$ band observation of the field. The correspondences are very good between the original and the kriged values for every intensity of the injected artificial sources, although increasing intensity means bigger area on which the point sources will spread, which increases the error in estimating the foreground properly.

\section{Conclusion}

Kriging has proven its effectiveness in removing point sources from even observations of the densest molecular clouds.

Surprisingly the accuracy increases with increasing density. This may be due to the much higher signal-to-noise ratio at the Taurus region with high FIR surface brightness (note the high ISM column density there).

\section{Acknowledgements}

This work was supported by the Hungarian OTKA NN-111016 grant.

\section{References}

Bagoly, Z., Szécsi, D., Balázs, L. G., et al. 2016, A\&A, 593, L10

Cressie, N. 1992, 1992TeNov...4..613C

Fehér, O., Tóth, L. V., Ward-Thompson, D., et al. 2016, A\&A, 590, A75 